\title{
REPLY TO A.W. OVERHAUSER "NOTE ON THE BAND THEORY OF MAGNETISM"
}

\author{
D. C. MATTIS
}

IBM Research Center, Yorktown Heights, New York

One of the main points of my article was that a purely spatial force has, and should have, little or no effect on the magnetic properties of electrons whereas interband Hund's-rule type exchange has strong and immediate magnetic consequences. The exactly soluble model fully confirmed this point, and if this makes it "magnetically impotent" then so be it! (Note that Overhauser's own model yields a contribution to the spin susceptibility, by the spatial forces, which vanishes as exp- $2 k_{F}$ in the limit of large $k_{F}$.) I believe that in three dimensions also, the spatial forces (long-range Coulomb repulsion, for example) play no determining rôle in the theory of magnetism, whereas the atomic exchange potentials among electrons of degenerate bands will determine whether or not a material has nontrivial magnetic properties, in competition with the "kinetic energy" associated with the band widths. The soluble model did not exhibit such properties of a three dimensional systems as long range order, or a Kohn effect (nonanalytic behavior of the response functions at $q=2 k_{F}$ ), and may be unrealistic in other respects, but it is the only soluble model that we have. Aside from this, if I may quote Faraday, "even to myself my ideas on this point appear only as the shadow of a speculation, or as one of those impressions on the mind which are allowable for a time as guides to thought and research."

Another main point of the article was that Hartree-Fock (H-F) theory, or perturbation theory, would predict the Pauli spin susceptibility wrong, among its other defects. I supported this conjecture by an unfortunate calculation, in which holes in the negative-energy Dirac sea were neglected. This neglect proved to be unjustified, as overhauser has quite properly noted, for it caused certain exchange integrals to depend spuriously on $k_{F}$. The following is a correct $\mathrm{H}-\mathrm{F}$ calculation: consider $H_{0}+H_{1}$, equations (II.3) and (II.4) of my paper, and set

$$
\rho_{j m}(p)=\sum a_{j, k+p, m}^{*} a_{j, k, m} \quad(j=1,2 \text { and } m= \pm 1 / 2)
$$

and

$$
\begin{aligned}
& n_{1, m}(k)=a_{1, k, m}^{*} a_{1, k, m}= \begin{cases}1 & k<k_{F 1 m} \\
0 & k>k_{F 1 m}\end{cases} \\
& n_{2, m}(k)=a^{*}{ }_{2, k, m} a_{2, k, m}= \begin{cases}1 & k>k_{F 2 m} \\
0 & k<k_{F 2 m}\end{cases}
\end{aligned}
$$

Then instead of equation (V.1), the diagonal Hamiltonian is:

$$
H_{D}=\sum_{k, m}\left(k n_{1 m}(k)-k n_{2 m}(k)\right)+\frac{\lambda}{2 L} \sum_{m, p, j} v(p) \sum_{k=-\infty}^{+\infty}
$$




$$
\left\{X \quad n_{j m}(k)\left(1-n_{j m}(k+p)\right)\right\}
$$

and the exchange energy is simply

$$
W_{\text {ex }}=\frac{2 \lambda}{\pi} \sum_{p>0} v(p)|p|
$$

This quantity is independent of the Fermi wavevectors, and therefore this H-F calculations shows the spin susceptibility to be independent of $\lambda$ in agreement with Overhauser's remarks and with the exact results. I am indebted to Drs. T.M. Rice and K. Sawada for providing the above derivation [1], which serves to correct the error in section $\mathbf{V}$ and to eliminate the troublesome "paradox". Drs. Rice and Sawada also pointed out that the exchange energy is just the leading term when the exact interaction energy [2] $W$,

$$
W=2 \sum_{p>0} p\left\{\left(1+\frac{2 \lambda v(p)}{\pi}\right)^{1 / 2}-1\right\}
$$

is developed in a power series in $\lambda$. This is a very significant observation, for what can be the meaning of $W_{e x}$ if such a power series does not exist? Fortunately, with the soluble model one can discover whether perturbation theory exists or not, merely by expanding the square root and examining the various integrals $I_{n}$ :

$$
I_{n}=\int_{0}^{\infty} d p p[v(p)]^{n} \quad \text { where } n=1,2,3, \ldots
$$

Each $I_{n}$ is proportional to the contribution of the $n$-th order diagrams. Perturbation theory has meaning only if all. $I_{n}$ are finite. But this is not the case for such singular potentials as the hard core or for any spatially long-ranged potential, for which $v(q)$ increases without bound as $q \rightarrow 0$. Consider for example, the class of potentials of the form

$$
v(p)=|p|^{-\alpha} \exp -\gamma|p|
$$

with $\alpha$ in the range $0<\alpha \leqslant 2$ and $\gamma$ a small positive convergence factor. The case $\alpha=2$ is quite analogous to the one-dimensional Coulomb potential (check this by Poisson's equation) and results in all $I_{n}=\infty$; whereas the exact energy is finite:

$$
\begin{gathered}
W=2 \sum_{p>0} p\left\{(1+2 \lambda p-2 \pi-1 \exp -\gamma p)^{3 / 2}-1\right\} \\
\left.\quad \underset{\left(\lambda \gamma^{2}\right.}{\longrightarrow} 1\right) \quad \frac{L}{2 \pi^{2}} \lambda \ln \frac{\text { const. }}{\lambda \gamma^{2}}
\end{gathered}
$$

Another example is reminiscent of the situation encountered by Gell-Mann and Brueckner [3] in their calculation for the three-dimensional Coulomb gas: choose $\alpha=1$, whereupon $I_{1}$ is finite (hence, so is $W_{\text {ex }}$ ) but all other $I_{n}$ diverge for $n \geqslant 2$. The exact energy $W$ is finite of course, but it just doesn't have a Taylor series expansion! What is the meaning of $W_{\text {ex }}$ in such a case?

Nevertheless, for weak and finite-range potentials the model no longer provides any valid counterexample against the use of perturbation theory or of H-F theory and I should like to 
amend my paper to this effect. I wish to thank Dr. D. Jepsen for a fruitful conversation, Drs. Rice and Sawada for their correspondence, and Dr. P.W. Anderson for the opportunity to respond to the criticism of my paper.

\section{REFERENCES}

1. Private communication.

2. Equation (A4) in Mattis and Lieb, J. Math. Phys. 6, 304 (1965), multiplied by a factor 2 to account for the Kramers degeneracy.

3. M. GELL-MANN and K. BRUECKNER, Phys. Rev. 106, 364 (1957). 